of the original text is retained and the original page numbers are inserted in the margin, so that reference to the original is easy.

The Introduction by Professor Braithwaite is almost as long as Gödel's paper; it is accurately and precisely written, primarily with the needs of a philosophical logician in mind. The Introduction begins by explaining the state of our knowledge of metamathematics at the time when Gödel's paper was written, and this is followed by accounts of arithmetisation, recursiveness, the " unprovability " theorem, and consistency. The final section deals with the syntactical character of Gödel's theorems.

There are accounts of Gödel's theorems in a number of books on mathematical logic. Thus this book will be valuable to mathematicians as a readily accessible source of the original paper. The Introduction by Professor Braithwaite is an account of Gödel's paper from a philosopher's point of view. The reviewer's reaction to this is perhaps what one would expect of a mathematician. The Introduction (especially the first, second, and final sections) is clear and informative, but in places one feels that a more mathematical approach is preferable.

R. M. DICKER

BURKILl, J. C., A First Course in Mathematical Analysis (Cambridge University Press, 1962), vi+186 pp., 22s. 6d.

This course is based on the idea of a limit and is intended for students who already have a working knowledge of the calculus. The analytical treatment of the calculus includes chapters on the Riemann integral and on differentiation of functions of several variables, but not on multiple integrals; the chapters on sequences and series exclude uniform convergence, upper and lower limits, and the general principle of convergence. There are examples after each section, and notes on these are given at the end of the book.

The author makes skilful use of informal explanations, and in general the ideas are presented extremely clearly and at a well chosen level. Thus the discussion of real numbers in the first chapter leads in a natural way to a statement of Dedekind's theorem as an axiom, and this is used to prove the existence of the supremum and infimum of a bounded set. More than average ability is necessary to follow the proofs of theorems, however, as steps have sometimes been omitted which might well have been included in an elementary textbook. Occasionally there is a serious gap; for example, the author does not prove that a convergent sequence is bounded. (This result is assumed on page 30; after the discussion of the sequence $1 /(n-10)$ on page 24 , the reader may doubt whether it is true).

These reservations are small, however, and it is a pleasure to be able to welcome a book on analysis written by an author who has a sense of style and who avoids the excessive use of symbolism which can make the subject unnecessarily difficult for the student.

\title{
P. HEYWOOD
}

DITKIN, v. A. AND PRUDNIKov, A. P., Operational Calculus in Two Variables and its Applications, translated by D. M. G. WISHART (Pergamon Press, 1962), $\mathrm{x}+167$ pp., $50 \mathrm{~s}$.

This book contains an account of the operational calculus in two variables based on the two-dimensional Laplace Transform,

$$
F(p, q ; a, b)=\int_{0}^{a} \int_{0}^{b} e^{-p x-q y} f(x, y) d x d y .
$$

The authors divide the text up into two distinct parts-the first part containing the 
basic theory and applications, and the second containing a useful list of transforms and their inverses. Throughout the text, it is assumed that the reader has had a good deal of experience with the operational calculus in one variable. The notations used in the book are those of Continental Europe, and the reader accustomed to the Anglo-American usage must continually consult the list of notations given in Part II.

Part I consists of two chapters. Chapter 1 deals entirely with the definitions and properties of the two-dimensional Laplace transform. In the second chapter, the authors state the basic definitions and prove some of the important theorems of the operational calculus in two variables. Also, some of these results are applied both to the evaluation of certain definite integrals and to the solution of some partial differential equations. The authors base their discussions on the Laplace-Carson transform,

$$
F(p, q)=p q \int_{0}^{\infty} \int_{0}^{\infty} e^{-p x-q y} f(x, y) d x d y .
$$

They show that it is a simple matter to prove that the relations which hold for the Laplace transform of Chapter 1 also hold for this transform. The authors illustrate the basic theory in the last section of the second chapter by using this transform to find solutions of some first order partial differential equations, the equation for the vibrating string, the heat-conduction equation, and Laplace's equation. An excellent list of formulae is given in the second part of the text.

On the whole the book is well written. However, one should observe that it is written primarily for the reader who wishes to apply the operational calculus in two variables, rather than for those who are interested in a more rigorous treatment of the subject.

M. LOWENGRUB

SCHWERdTFEger, H., Geometry of Complex Numbers (Oliver and Boyd, Edinburgh, 1962), $x i+186$ pp., 30s.

One way of introducing complex numbers into geometry is to admit them as individual coordinates of points, thus obtaining a complex geometry. Alternatively, the real and imaginary parts of a single complex number may be used as the real coordinates of a point in a plane, the familiar Argand diagram or Gauss plane of elementary mathematics. The second approach forms the topic of the present book so that, as we are warned in the Introduction, the geometry is essentially real, though "imaginary circles" have to be considered in so far as they define real inversions.

The first of the book's three long chapters deals with the analytic geometry of circles, which are represented by hermitian matrices, with stereographic projection and cross-ratios. Chapter II gives a very comprehensive account of the properties and classification of Moebius transformations. In the final chapter these results are applied to hyperbolic, spherical and elliptic geometry. The author's aim is to broaden the student's view of the relations between modern algebra, the geometrical properties of complex numbers and other geometrical theories. That students should be discouraged from assigning knowledge to water-tight compartments is most praiseworthy, but the reviewer wonders whether time could be found in the average course for so detailed a discussion of the book's specialised topic. Those who are interested in such an exhaustive treatment will, however, undoubtedly find it useful.

Some points of minor detail might perhaps have been treated more economically. a somewhat simpler construction for the fourth harmonic of $z_{3}$ with respect to $z_{1}$ and $z_{2}$ (page 38) is to join $z_{3}$ to the point of intersection of the tangents at $z_{1}$ and $z_{2}$ to the circle through the three points; the second intersection of the line and the circle is the point required. If the condition for a Moebius transformation to be an 